\title{
Surface Acoustic Wave Vibration Sensor Electronic System
}

\author{
J. Filipiak ${ }^{a, *}$, L. SOlARz ${ }^{b}$ AND G. SteCZKO ${ }^{a}$ \\ ${ }^{a}$ Institute of Electronic and Control Systems, Technical University of Częstochowa
}

Armii Krajowej 17, 02-240 Częstochowa, Poland

${ }^{b}$ Department of Civil Engineering, Military University of Technology, S. Kaliskiego 2, 00-908 Warsaw, Poland

The paper presents surface acoustic wave vibration sensor electronic system. The system cooperates with surface acoustic wave delay line. The sensor has been designed for electronic warning systems. The sensor is a four-terminal network. In the system, there is a cascade connection of sensors by means of coaxial cable. Test signal and constant supply voltage are jointly sent between sensors. Separating the signals, their summation and amplification of test signal as well as matching the line to impedance $50 \Omega$ are performed by surface acoustic wave vibration sensor electronic system. The electronic system devices and the making of them are discussed. System development of such a sensor is presented and its design is analysed. Experimental examination results of surface acoustic wave vibration sensor are presented. Parasitic effects occurring in vibration sensor electronic system operation connected with electromagnetic coupling and ground current coupling are discussed.

PACS: 43.25.Fe, 77.65.Dq, 68.35.Iv

\section{Introduction}

Surface acoustic waves (SAW) propagate over the solid body surface bordering on gas or vacuum. In technical applications, SAW are induced by interdigital transducers (IDT) made on piezoelectric plates. A system of two cooperating IDTs constitutes a band-pass filter. The filters operate from several $\mathrm{MHz}$ to $2.5 \mathrm{GHz}$. They are wattless devices. They bring loss from several to several tens of $\mathrm{dB}$.

Surface wave availability in SAW filters enables to apply them as sensors of various physical quantities [1-7]. A change in measured physical quantity causes a change in SAW propagation time. The principle of operation of the sensors consists in measuring a delay change in SAW filter. SAW filter plays the role of SAW delay line. The delay line operates on one carrier frequency. The distance between transducers determines the delay time of SAW delay line. Introduction of an oscillating motion of the plate changes the delay time of SAW delay line. This enables to construct SAW vibration sensors.

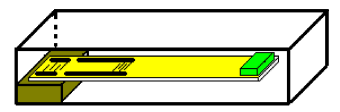

Fig. 1. SAW vibration sensor design.

Figure 1 shows the design idea of SAW vibration sensor. The basic element of the sensor is a plate made of piezoelectric material. One side of the plate is stiffly attached to sensor casing, while to the other free side there may be attached seismic mass. On the upper surface of the plate, SAW delay line in form of a four-terminal network has been made. Four-terminal network is a

\footnotetext{
* corresponding author; e-mail: gem@gemsc.com.pl
}

narrowband electric filter with appropriately high mid-frequency.

Motion of sensor casing generates vibrations of its plate and alteration in the delay of SAW delay line. It is a cause of alteration in a high frequency signal phase which goes through such a line. The volume of signal phase alteration will be proportional to alteration in the delay of SAW delay line. Frequency of phase change will be equal to vibration frequency of the sensor plate. The sensor plate must have one natural vibration frequency [8]. The amplitude of high frequency signal phase changes will be proportional to the amplitude of vibrations of the sensor plate. These properties may be used to determine motion of substrate to which the sensor casing is fixed and to identify the sensor.

The paper [8] presents SAW vibration sensor for an electronic warning system. Cascade connection of such sensors enables to construct a warning system. Figure 2 presents a block diagram of a warning system with SAW vibration sensors.

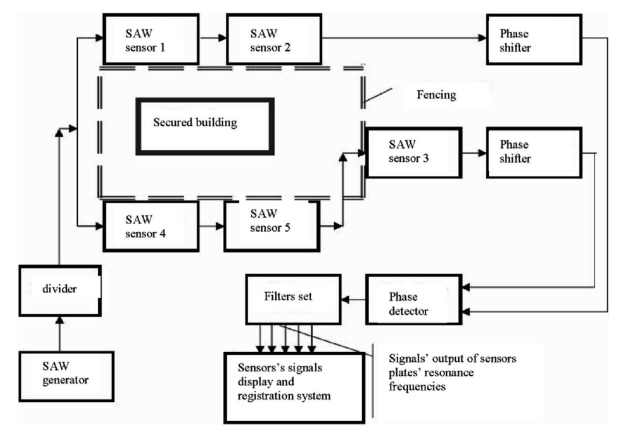

Fig. 2. A block diagram of a vibratory warning system with SAW sensors.

The system consists of five SAW vibration sensors. Each sensor has a different plate resonance frequency. Sensors are joined in a cascade connection in two lines. 
SAW generator is a source of $74 \mathrm{MHz}$ test signal. The test signal is divided into two lines. Each line goes through SAW vibration sensors joined in a cascade connection. Circuit shown in Fig. 2 consists of components cooperating and interacting with each other only electronically. Mechanical displacements of sensors are independent, they change the test signal phase, namely the electrical parameters of the circuit. For easy assembly, the measurement and supply signals are sent through one coaxial cable. For easy extension of the system by additional sensors, losses created by SAW delay line must be compensated by an amplifier cooperating with SAW delay line. Figure 3 shows a cascade connection of additional sensors. SAW delay line, being a part of the sensor, consists of two IDTs. It is made on a ST-cut quartz plate because of its good temperature properties.

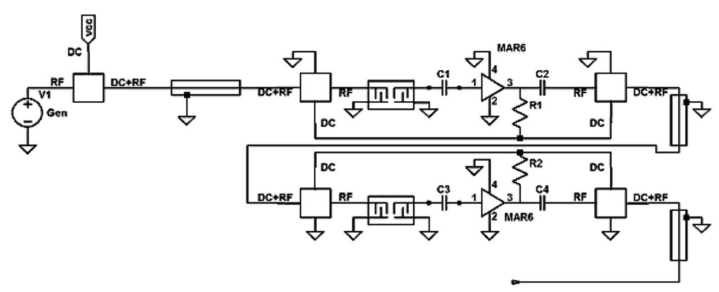

Fig. 3. Cascade connection of SAW vibration sensors.

The above-mentioned assumptions are requirements which must be fulfilled by an electronic system of SAW vibration sensor. The making of electronic components constituting the vibration sensor will be discussed in the next section.

\section{Vibration sensor electronic components}

In order to ensure the transmission of test and supply signals through one coaxial cable there must be a system at the sensor input separating the test signal $(74 \mathrm{MHz})$ and supply signal $12 \mathrm{~V}_{\mathrm{DC}}$ (separator). At the output there must be a system summing up the test signal with a constant supply voltage (adder). Test signal (high frequency) after going through SAW delay line must be amplified to input quantity. It will ensure loss compensation caused by SAW delay line. In the entire line of high frequency test signal $(74 \mathrm{MHz})$ a characteristic impedance of $50 \Omega$ should be retained. Input and output impedance must have the value of $50 \Omega$. Figure 4 shows the basic functional elements of SAW vibration sensor. Depending on the function in the whole system the following components may be distinguished:

1. system separating and summing up the test and supply signals;

2. systems adjusting the impedance of SAW line to $50 \Omega$;

3. SAW delay line;

4. amplifier compensating losses caused by SAW delay line.
The method of making the aforementioned components will be discussed in the next section.

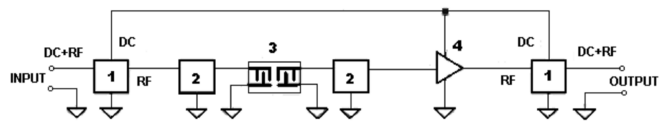

Fig. 4. Block diagram of SAW vibration sensor.

\subsection{System separating or summing up electrical signals (separator/adder)}

A system separating test and supply signals is placed at the sensor input. A system summing up these signals is placed at the sensor output. Figure 5 shows a system separating or summing up test and supply signals.

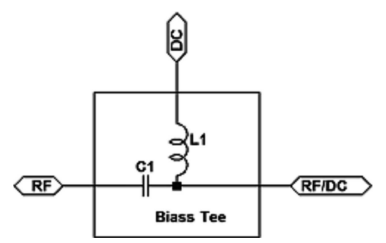

Fig. 5. System separating or summing up test and supply signals.

The system is in a form of a circulator. It is connected to the line of high frequency signal with a characteristic impedance of $50 \Omega$. A point of separation (or summation) of signals is the place where additional impedance is added to a line of characteristic impedance of $50 \Omega$ by inductance $L_{1}$. It may change the characteristic impedance of the line and be the reason of signal reflections. In order to avoid this the quantity of added impedance must be much larger than line characteristic impedance $(50 \Omega)$. In order to fulfil this requirement inductance $L_{1}=4.7 \mu \mathrm{H}$ of its own parallel resonance frequency of $74 \mathrm{MHz}$ has been chosen. Figure 6 shows inductance equivalent system diagram.

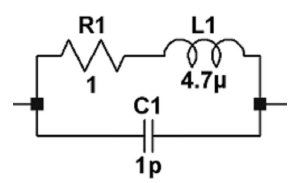

Fig. 6. Inductance equivalent system diagram.

In reality, the chosen inductance is a parallel resonant circuit. Figure 7 presents change in impedance of such a system in frequency function.

For frequency equal to $74 \mathrm{MHz}$ the system impedance value amounts to $400 \mathrm{k} \Omega$. It is relatively high in comparison with characteristic impedance of the test signal transmission line $(50 \Omega)$. It is then possible to obtain considerable attenuation of the test signal entering the supply circuit and it practically eliminates reflections at 


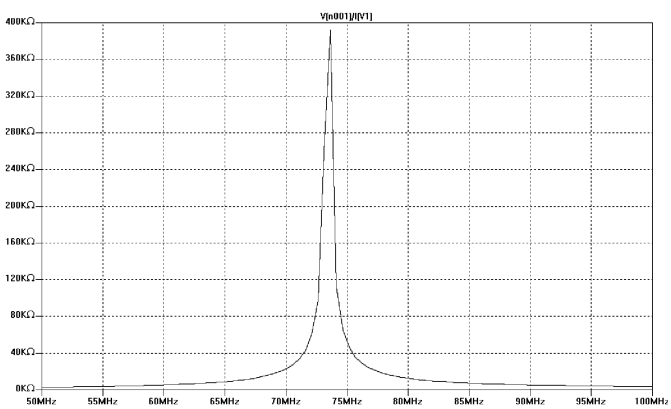

Fig. 7. Relationship between impedance and frequency of a system presented in Fig. 6 .

the point of signal separation or summation. Figure 8 presents an actual system separating the test and supply signals. Impedance of connected in series: C1 capacity and RF output impedance is equal to characteristic impedance of $50 \Omega$. A diagram presented in Fig. 8 enables to analyze test signal attenuation in supply circuit. It allows calculating the change in line characteristic impedance made by the separating system.

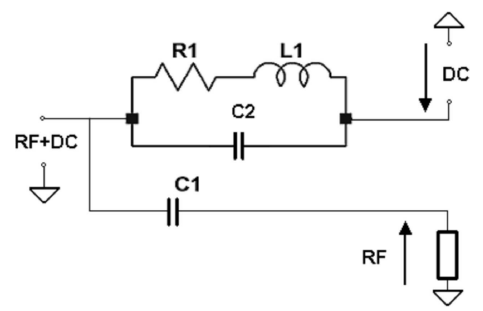

Fig. 8. Actual system separating the test and supply signal.

Figure 9 shows attenuation of the test signal at DC output and change in line impedance in frequency function for $R 1=1 \Omega$.

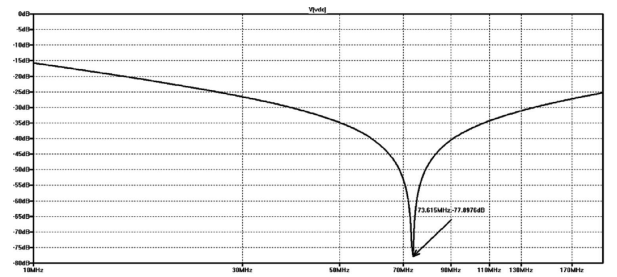

Fig. 9. Attenuation of test signal at DC output in frequency function.

Calculations of transmission line impedance change have been done on the assumption that the test signal line has the impedance equalling $50 \Omega$ in the entire frequency range. This assumption is correct in the range of line frequency. It substantially simplifies modelling of the system (Fig. 10).

This simplification does not influence the results in the system frequency range. For the sensor frequency

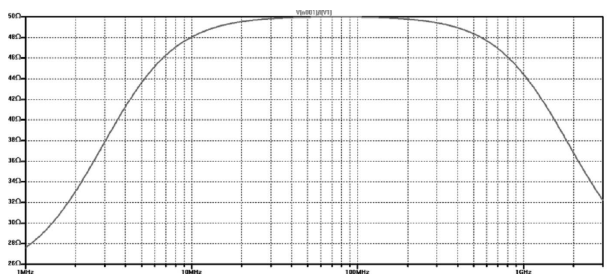

Fig. 10. Change in test signal line characteristic impedance in frequency function.

equalling $74 \mathrm{MHz}$ the test signal attenuation at DC output equals $-78 \mathrm{~dB}$, and line impedance equals $50 \Omega$. A separating or summing up system prepared in such a manner does not influence the test signal transmission through SAW vibration sensor.

\subsection{Matching system for interdigital transducers}

Systems matching IDTs to the impedance of cooperating electronic systems in a given frequency are widely discussed in literature [9-14]. During the making of SAW vibration sensor electronic system two different systems matching SAW delay line and the impedance of $50 \Omega$ have been made. Inductance has been used in the first system [10]. In the second system, a matching system for an IDT has an additional transformer which enables its symmetrical supply [11].

In the first type, the systems matching SAW line impedance to $50 \Omega$ are identical for both IDTs. A matching system has been discussed in [10]. Figure 11 presents transducer matching system.

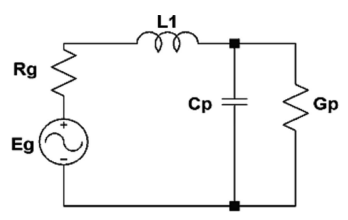

Fig. 11. Matching system for IDT to impedance of $50 \Omega$.

A matching element of a transducer of conductance $G_{P}$ and capacity $C_{P}$ to impedance $R_{\mathrm{g}}=50 \Omega$ is inductance $L_{1}$. Matching requires separating at transducer conductance $G_{P}$ of available power taken from supply source $E_{\mathrm{g}}$ of internal impedance $R_{\mathrm{g}}=50 \Omega$. This requirement is fulfilled for inductance value determined by [10]:

$$
L_{1}=\frac{C_{P}}{G_{P}^{2}+\omega^{2} C_{P}^{2}} .
$$

For frequency equalling $74 \mathrm{MHz}$ transducer conductance equals $0.74 \mathrm{mS}$, and the capacity equals $3.13 \mathrm{pF}$. These quantities have been used to calculate the inductance value $\left(L_{1}=900 \mathrm{nH}\right)$, for which transducer matching requirement to impedance of $50 \Omega$ has been fulfilled. Matching of IDT to impedance of $50 \Omega$ has been done by measuring reflection coefficient. Figure 12 shows 
change in reflection coefficient quantity in frequency function from matched transducer in a system of impedance of $50 \Omega$.

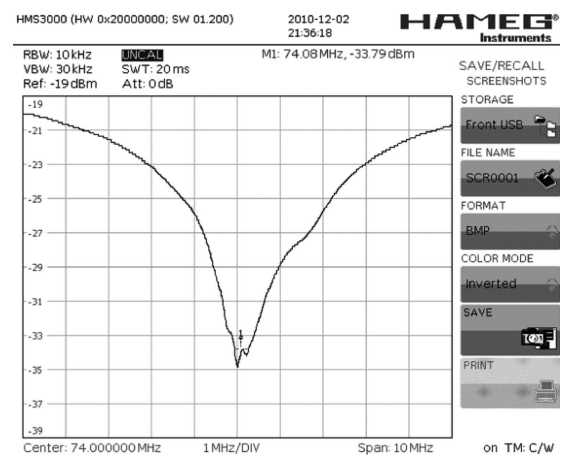

Fig. 12. The relationship between reflection coefficient and matched transducer in frequency function.

In the second manner of matching one transducer has been matched as described above. The second transducer has been matched in a system presented in Fig. 13.

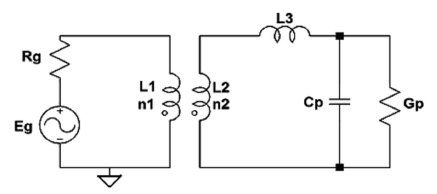

Fig. 13. Symmetrical matching of transducer with the use of transformer.

Introducing a transformer changes inductance value L3. Parameters of matched transducers and the volume of line losses are identical as in the first type of matching. Transformer primary function is to bring a symmetrical supply of one IDT and to reduce the strength of a signal which goes directly through SAW delay line transducers.

\subsection{Amplifier}

The role of an amplifier is to compensate losses caused by SAW delay line. An amplifier has been built on a monolithic system MAR-6 manufactured by Mini-Circuits. Figure 14 shows a diagram of an amplifier being a part of an electronic system of SAW vibration sensor.

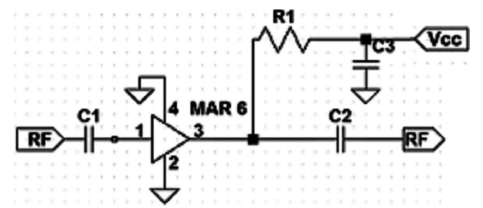

Fig. 14. A diagram of an amplifier for SAW vibration sensor.

The amplifier is supplied at the output side by an R1 resistor. The $\mathrm{R} 1$ value is selected according to the $\mathrm{DC}$ supply voltage. For a supply voltage of $12 \mathrm{~V}$ the resistance R1 equalled $560 \Omega$. An amplification of $22 \mathrm{~dB}$ was achieved for test signal frequency of $74 \mathrm{MHz}$. It is the highest amplification value possible to achieve in this system. The value of current input equalled $16 \mathrm{~mA}$. Figure 15 shows experimental frequency characteristics of SAW vibration sensor.

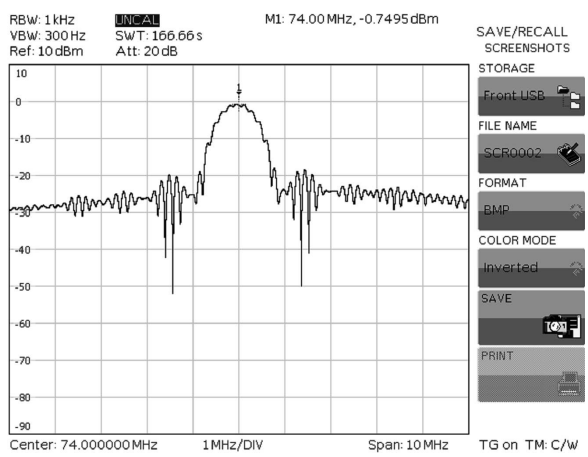

Fig. 15. Attenuation frequency diagram of SAW vibration sensor.

Measurement of attenuation frequency diagram of SAW vibration sensor has been conducted on a spectrum analyser HMS 1010. A supply voltage system has been put at line input. A supply voltage blocking system has been put before the analyser at line output. Losses of $0.75 \mathrm{~dB}$ consist of line losses and losses in the connection wiring and discussed separation systems. The value of these losses has been estimated at the level of $1 \mathrm{~dB}$. A conclusion may be drawn that an amplifier compensates the losses caused by SAW delay line.

A theoretical shape of attenuation frequency diagram of a sensor should be described by the function $\sin (x) / x$. An experimental characteristic has high-frequency irregularities. Their reasons are the signals going from sensor output to input, omitting sensor electronic components. This signal amplitude is around $-36 \mathrm{~dB}$ lower than useful signal amplitude. The reason of occurrence of signal going from sensor input to output will be discussed in the next section.

\section{Parasitic signals}

Parasitic signals are the signals going from electronic system input to output, omitting any component which is a part of the test signal transmission line. It is possible due to the occurrence of a parasitic coupling between any places of electronic system. There are two mechanisms leading to the occurrence of couplings [15]. The first one is electromagnetic coupling. The second one is ground current coupling. Figure 16 shows the mechanism of electromagnetic coupling.

Red lines indicate paths of electromagnetic coupling which may occur in the electronic system of SAW vibration sensor. Electromagnetic couplings occur in all the electronic components constituting a sensor system. Paths of printed circuit are matched to the impedance of 


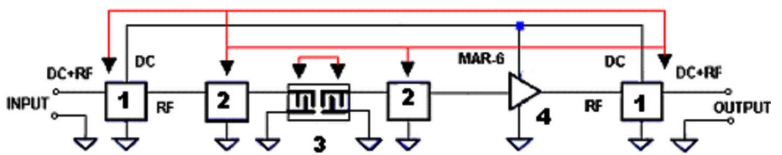

Fig. 16. Electromagnetic coupling in electronic system of SAW vibration sensor.

$50 \Omega$. They are simultaneously transmitting and receiving aerials. Their efficiency depends on the path length. A similar role is played by inductances occurring in the system and capacities between paths. In order to reduce the electromagnetic coupling the inductances should be placed perpendicularly to each other and placed at a distance. These elements define the manner of making of the printed circuit plates. Any problems are solved individually, in accordance with a chosen construction. The fundamental problem is an occurrence of electromagnetic coupling between the transducers and SAW delay line. Bus-bars delivering electric signal to transducers are placed on an immobile part of the plate. They are long and they are placed close to each other. It causes an increase in capacity between IDTs. The direct signal going through this way is also strengthened. Because of sensor plate motion its casing is larger than those used in traditional SAW filters. It also causes an increase in direct signal strength in the delay line. This problem and possible solutions are known in literature [10-14]. The most effective solution is symmetrical supply of one of transducers and their functioning in a bridge circuit. Such a solution has been used in the presented SAW vibration sensor. The signal strength at the level of $-35 \mathrm{~dB}$ has been achieved.

The second mechanism causing increase in direct signal strength is the ground current coupling. Figure 17 shows the mechanism of this coupling.

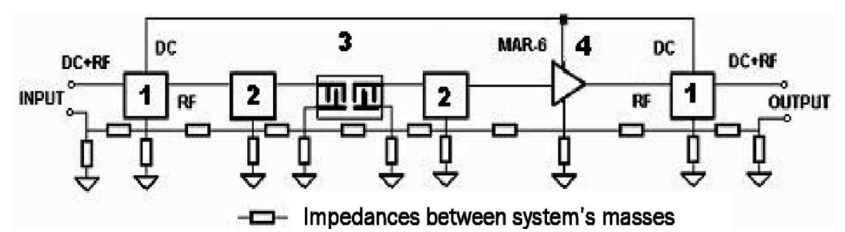

Fig. 17. The mechanism of ground current coupling.

Ground current couplings occur only when the connection between component mass and joint mass is not perfect. A diagram in Fig. 17 shows this effect by introduced impedances. An ideal connection is characterised by a null value of all impedances. Introduced impedances change current distribution in the entire system.

Values of these impedances are small (fractions of ohm). That is why they are difficult to model. A physical making of component mass connection to the system joint mass must be considered during the design stage of the system. Reduction of this value by careful preparation of the system joint mass is a proper solution. Sim- ilarly to the first mechanism, SAW delay line plays an important role. Impedance of bus-bars and impedance of contacts leading the signal to transducer are crucial elements in SAW vibration sensor delay line.

The joint mass of the discussed system has been made of $5 \mathrm{~mm}$ copper plate to which a printed-circuit board has been soldered. This side of the plate was completely bonded. The electronic system joint mass has been connected to component masses. Figure 18 shows SAW vibration sensor.

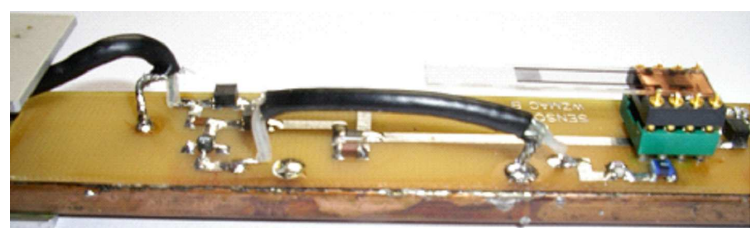

Fig. 18. SAW vibration sensor.

Only supply voltage of the amplifier system goes through printed circuit paths. Longer segments of test signal line have been made by means of coaxial cables. It allowed to reduce an electromagnetic couplings value in the system. Attenuation frequency diagram of this sensor is presented in Fig. 15. Figure 19 presents this characteristic after a shut-down of amplifier supply.

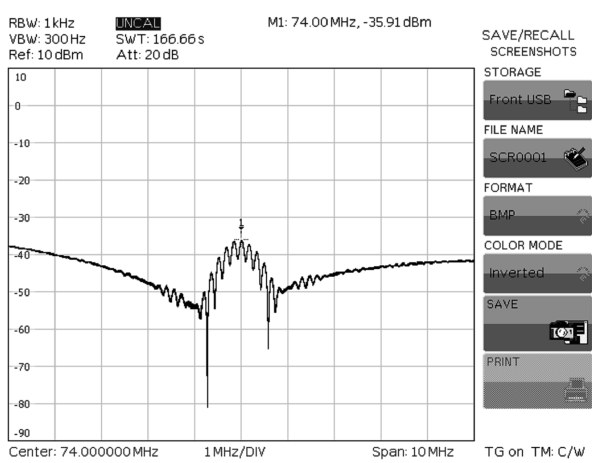

Fig. 19. Attenuation frequency diagram of SAW vibration sensor after shut-down of amplifier supply.

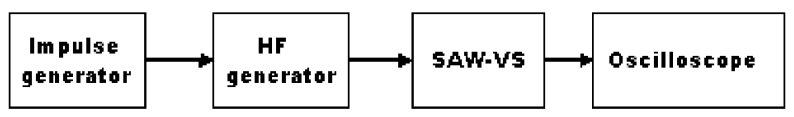

Fig. 20. Meter circuit for (parasitic) signals in SAW delay line.

Shapes of characteristics in delay line operation band are similar in both figures. It suggests that signal source at the sensor output is situated behind SAW delay line. This signal strength is $-35 \mathrm{~dB}$ below the sensor frequency characteristic signal. Beyond the operation band the signal strength equals $-40 \mathrm{~dB}$. Conducted measurements make use of the time trace of stationary signals. The 
lack of information about the delay of these signals does not allow to determine their source. IDTs are selectively matched to the impedance of $50 \Omega$ (Fig. 12). It can be a signal going directly between interdigital transducers of SAW delay line. In order to explain the origin of the signal, it is important to learn about its delay time. It has been made in a system presented in Fig. 20 .

At sensor input a signal in form of wave packet at frequency of $74 \mathrm{MHz}$ has been delivered. The length of the packet is smaller than line delay. In this way a temporary separation of the direct and useful signals has been ensured. Figure 21 presents timing of signals at the output of SAW vibration sensor. There are five signals at the sensor output. The first signal is going directly from sensor output to its input. The second one is a useful signal. Next three signals are reflected from the plate edge. Reflected signals will be attenuated by a damping paste.

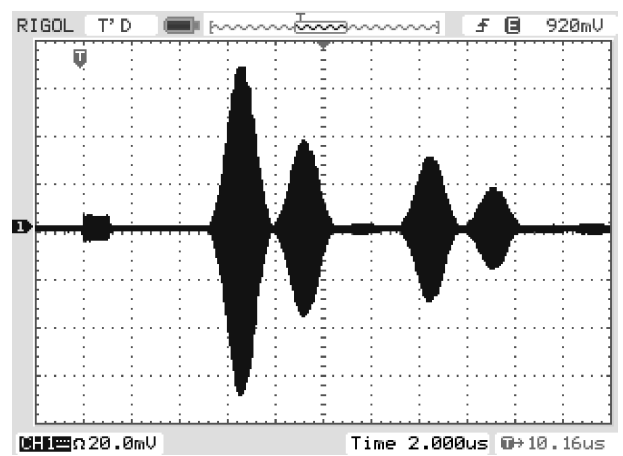

Fig. 21. Time run of signals in SAW delay line.

The amplitude of direct signal is $-35 \mathrm{~dB}$ below the useful signal. It leads to conclusion that the signal shown in Fig. 17 is a signal going directly between SAW delay line IDTs. When the electronic system is properly made, the elimination of this signal is the most fundamental problem in SAW vibration sensor design.

\section{Conclusion}

This paper presents a SAW vibration sensor electronic system. The sensor has been designed and manufactured in a form of a four-terminal network. The sensor can be joined in a cascade connection by means of a coaxial cable with different sensors of the same type. Test signal and constant supply voltage are jointly sent between sensors. The electric system is responsible for: separating signals, summing up signals, amplifying the test signal and matching the line to the characteristic impedance of a slotted line. The electronic system components and their functions are discussed. A cooperation of neighbouring components leads to their mutual interaction. It leads to a change in their parameters. For example, a capacity of system separating signals changes the value of inductances important for matching an IDT. That is why an electronic system of SAW vibration sensor should be manufactured as a whole, not in a form of successively discussed components.
The results of experimental examinations of parameters of SAW vibration sensor are presented. Parasitic effects which occur in running of vibration sensor electronic system connected with electromagnetic coupling and ground current coupling are discussed. The most fundamental problem in SAW vibration sensor design is elimination of a signal going directly from sensor input to its output. It has been shown that careful preparation of the sensor electronic system helps in eliminating parasitic signals coming from ground current couplings.

However, the most fundamental problem is strength of the signal going between input and output of SAW delay line. The reduction of its strength is one of the most fundamental problems occurring in creation of SAW delay line and electronic systems directly cooperating with this line. The electronic system has been prepared for SAW vibration sensors used in an electronic warning system [9].

\section{Acknowledgments}

This work was supported by the Polish Ministry of Science and Higher Education as a project "Vibration Warning System with SAW Vibration Sensors" included in the scientific budget of 2009-2011.

\section{References}

[1] H. Wohltjen, R. Dessy, Anal. Chem. 9, 1458 (1979).

[2] C. Cullen, T. Reeder, in: Proc. Ultrasonics Symp., San Diego, CA Tech. Doc. 271, San Diego 1975, p. 519.

[3] B. Drafts, Sensors 10, 1 (2000).

[4] D.S. Ballantine, R.M. White, S.J. Martin, A.J. Ricco, E.T. Zellers, G.C. Frye, H. Wohltjen, Acoustic Wave Sensor - Theory, Design, and Physico-Chemical Applications, Academic Press, San Diego 1997.

[5] J. Filipiak, C. Kopycki, Sensors Actuators 76, 318 (1999).

[6] J. Filipiak, L. Solarz, G. Steczko, Mol. Quant. Acoust. 28, 71 (2007).

[7] B. Pustelny, T. Pustelny, Acta Phys. Pol. A 116, 383 (2009).

[8] J. Filipiak, L. Solarz, G. Steczko, Acta Phys. Pol. A 116, 302 (2009).

[9] J. Filipiak, L. Solarz, G. Steczko, Electrical Rev. 11a, 177 (2010) (in Polish).

[10] J. Filipiak, Surface Acoustic Wave Acceleration Sensors, Vol. 121, Technical University of Częstochowa, Częstochowa 2006, p. 1.

[11] E. Danicki, J. Filipiak, Electronics 22-26, 10 (1982) (in Polish).

[12] A.A. Oliner, Acoustic Surface Waves, Springer Verlag, Berlin 1978.

[13] L. Matthews, Surface Wave Filters, Wiley, New York 1997.

[14] P. Morgan, Surface Wave Devices for Signal Processing, Elsevier, London 1985.

[15] www.saw-devices.com . 